\title{
Continuous Fitness at Home: Designing Exercise Equipment for the Daily Routine
}

\author{
M.C. Gaspar ${ }^{1}$, F. Ventura ${ }^{2}$, C. Pereira ${ }^{3}$, C.M. Santos ${ }^{4}$ \\ 'Departamento de Engenharia Industrial, \\ Escola Superior de Tecnologia, Instituto Politécnico de Castelo Branco, \\ Av. do Empresário, 6000-767 Castelo Branco, Portugal. \\ calvete@est. ipcb.pt \\ ${ }^{2}$ Departamento de Engenharia Mecânica, \\ Faculdade de Ciências e Tecnologia da Universidade de Coimbra, \\ Pinhal de Marrocos, 3030-201 Coimbra, Portugal \\ Eernando.ventura@dem.uc.pt \\ ${ }^{3}$ Departamento de Engenharia Mecânica, \\ Instituto Superior de Engenharia de Coimbra, \\ Rua Pedro Nunes, 3030 - 199 Coimbra, Portugal. \\ candida@mai1.isec.pt \\ ${ }^{4}$ Escola Superior de Tecnologia da Saúde de Coimbra, \\ Rua 5 de Outubro, 3046-854 Coimbra, Portugal 1. \\ kaleu@sapo.pt
}

\begin{abstract}
The main goal of this work is to design tailored fitness devices that allow performing simultaneously home routine tasks and physical exercise, increasing the amount of physical activity in the daily routine. Physical therapists and design engineers have studied the most usual chores, as well as different types of training, to develop innovative and versatile solutions.
\end{abstract}

\section{Introduction}

The most recent recommendations indicate that people of all ages should include a minimum of half an hour of physical activity of moderate intensity on most, if not all, days of the week. It is also acknowledged that for the majority of people, health improvement can be obtained by engaging in physical activity of more vigorous intensity or of longer duration. Physical activity has numerous beneficial physiologic effects, e.g. on the cardiovascular and musculoskeletal systems, but benefits on the metabolic, endocrine, and immune systems performance are also considerable.

To maximize the benefits and results arising from workout it is necessary to perform a moderate amount of exercise on a regular basis. Exercising at home provides a convenient form of physical activity with the major advantage of maximizing time and effort. Nonetheless, conventional home gym fitness devices have several disadvantages such as the space required, their cost and the effective time spent when using them. On the other hand, they are rarely used by most persons, mainly due to the lack of motivation and the need to quit home routine.

Please use the following format when citing this chapter:

Gaspar, M. C., Ventura, F., Pereira, C., Santos, C. M., 2006, in IFIP International Federation for Information Processing, Volume 221, Human Work Interaction Design: Designing for Human Work, ed. T. Clemmensen, Campos, P., Omgreen, R., Petjersen, Al, and Wong, W., (Boston: Springer), pp. 147-160 


\section{Current Urban Culture}

In current urban lifestyle, daily life common physical tasks have been gradually replaced by machines and appliances. Yet, it is well known that one of the easiest ways of getting adequate exercise is to forego the use of an elevator or other mechanical conveniences and choose instead doing these activities manually, i.e. by one's own body. This type of exercise is fairly easy to do since it is purposeful and uncontrived.

\subsection{The Home Routine}

Not a long time ago, daily life was made up of a series of physical tasks. Stairs needed to be climbed, dishes and clothes needed to be washed by hand and a series of simple chores were done manually. Although modern progress has increasingly substituted physical activity by automated or mechanized devices, it is still possible to regain the home field to machines by doing manual chores during the daily schedule. As a consequence, performing manually useful household tasks can lead to a better body condition.

Physical exercise is part of one's life and refers to the walking, moving, and lifting inherent in just moving through one's daily schedule. In this work, household and leisure activities at home have been analysed on the pursuit of identifying significant time intervals that can be converted into physical exercise occasions. Figure 1 shows some of the most significant regular activities at home that take more than just simple instants to be performed. Although these activities are usually performed in a steady location with a somewhat rigid posture, they can easily be converted into a physical activity occasion if complemented by the use of proper fitness devices.

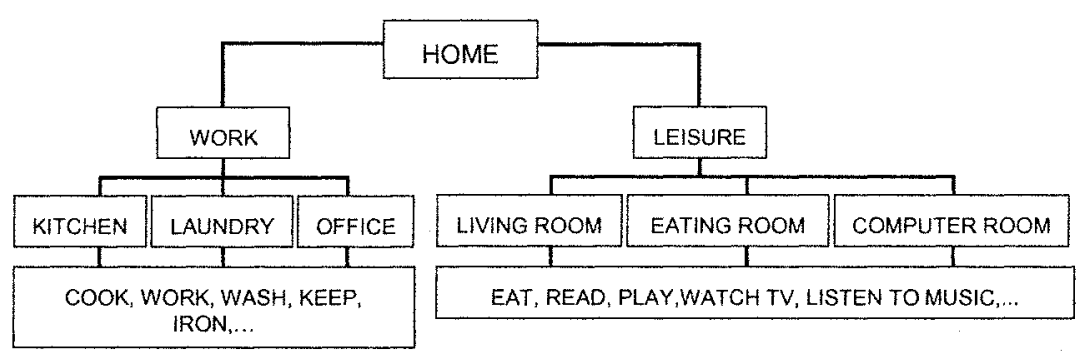

Fig. 1. Ordinary home routine repetitive tasks.

\subsection{Personal Health Care and the Need to Exercise}

In developed countries, due to sedentary lifestyle and labor-saving devices, many people do not get enough physical exercise. Few of today's occupations require vigorous physical activity and much of the leisure time is spent in stationary 
occupations. Thus, sedentary and obesity are considered two of the main modern plagues resulting from the lack of physical activity in the current urban habits. Although most of the population is concerned about its physical condition to improve health, looks or athletic performance, in practice they usually do not walk up the stairs instead of taking the elevator.

Because many people in developed countries also suffer from increased job and lifestyle stress, the lack of adequate exercise is doubly harmful. As a consequence, physical exercise is being recommended by almost all medical specialties and the benefits get well beyond the recovering of the musculoskeletal systems. Cholesterol lowering, vascular tonicity, cardiac muscle strengthening, enhancement of aerobic capacity and even beneficial physiologic effects are amongst the major benefits of physical exercise.

There are two types of exercise a person should do in order to stay healthy: aerobic exercise and resistance training. While aerobic exercise means doing something fast and repetitive enough to get one's heart beating $80 \%$ faster than normal, resistance training refers to lifting or pushing against something heavy or difficult to move. To obtain the best benefits from aerobic exercise, it should be performed in at least 20 minutes intervals. As such, when trying to adapt home routine into physical exercise, the selected tasks should take similar time intervals. However, considering that more than one household task can be transformed in an opportunity to do exercise, the cumulative time of different chores can have the same type of physical benefits.

\section{Conventional Home Fitness Devices}

To maximize the benefits and results from workout it is necessary to perform a moderate amount of exercise on a regular basis. Modern urban population spends most of its time between professional activity and home. The increasing work schedules and the unavoidable household tasks lead to a surprisingly short period of effective free time. Exercising at home provides a convenient form of physical activity with the major advantage of maximizing time and effort.

\subsection{Commercially Available Devices}

Current lifestyle does not permit that most people may walk to work or have a physically intense professional occupation. In such cases, it is necessary to set time aside to deliberately exercise. The need to exercise and the lack of free time turn into a dilemma, which people often solve getting a regular exercise program in any fitness academy. One alternative solution is the use of home fitness devices.

State-of-the-art home fitness devices with the objective of performing physical exercise indoor have several advantages, such as allowing exercise in a regular fashion, at any time of the day or night, and providing a safe and adequate home exercise for all kinds of people. There is a wide range of commercially available equipment devoted to exercise all major muscular groups. Most widely appreciated are steppers, cycles or elliptical trainers [Fig. 2]. 

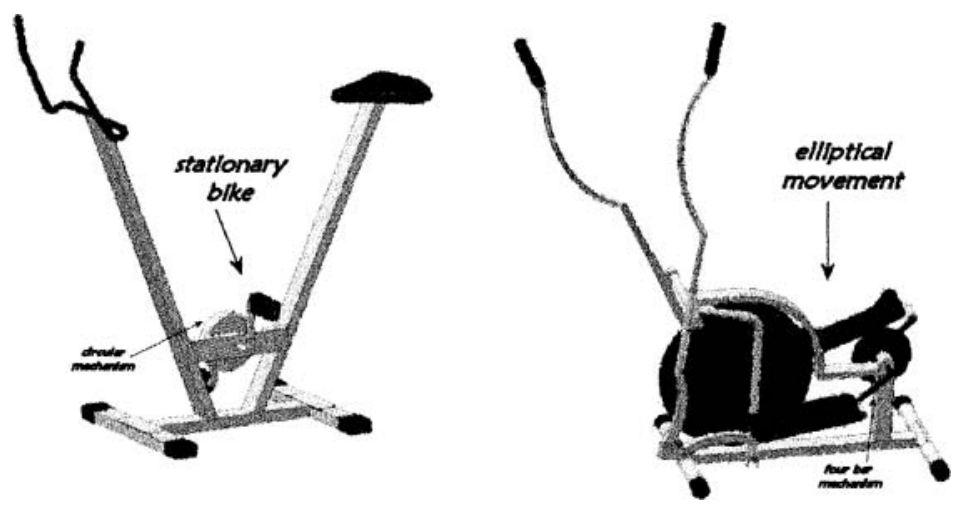

Fig. 2. Conventional home fitness devices.

Home gym equipment might also be a possible solution. Designed for the home, these gyms try to offer a wide variety of full range-of-motion exercise movement in a single group of devices. However, the large space required and the high costs associated are many times inhibiting factors for their widespread.

\subsection{Main Restrictions to the Use of Conventional Home Fitness Devices}

Very few people can enroll an exercise program for more than a few weeks. Most people are impatient and want to get more done in less time and with less effort. As such, if people are not well motivated, it is very unlikely that they will eagerly free some time on their personal schedule to quit other more rewarding occupations.

Even when fully motivated, the lack of space that characterizes most current urban constructions turn many times into an impossible quest the use of conventional home fitness devices. People have to face the problem of not only managing some space to perform the exercises [Fig. 3], but it is many times very difficult to find free space to keep the equipments when they are not in use.

Since each type of home fitness device is usually dedicated to exercise a specific muscles group, the need to exercise completely the whole body would turn the space problem much more evident. Home gyms try to offer a complete solution, but as referred, the space and cost associated are severe limitations on their use.

As a conclusion, the use of lightweight, compact and easy to move solutions would be a great improvement on the enhancement of home fitness devices. 

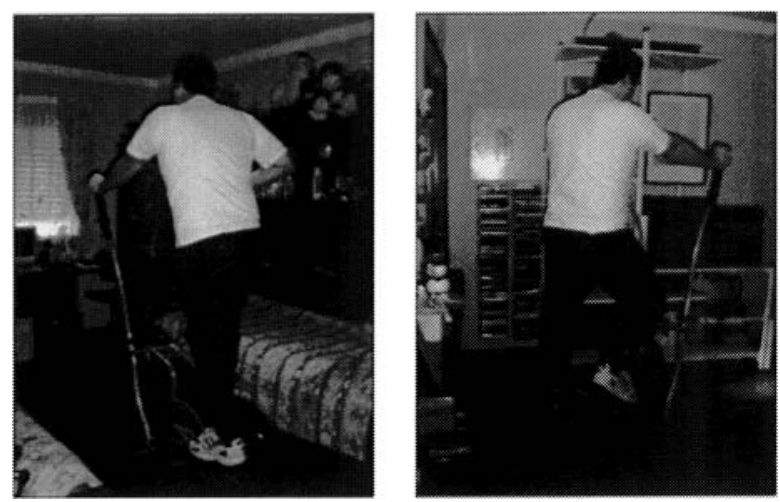

Fig. 3. Performing exercises on a home fitness device.

\section{Home Exercise Equipment for the Daily Routine}

It is well established that physical exercise is an effective need for current urban culture. However, the difficulties that arise every time a less motivated individual intends to carry out a regular exercise program show that this problem is far from being solved.

This work proposes a commitment between the basic need to undertake regular physical exercise and the lack of time that in general characterize a professional and social active person.

\subsection{Main Requirements}

As referred before, even the busiest person must perform at least a minimum home routine in order to keep order in personal life. Considering that an individual manages to motivate himself to carry out a physical exercise program at home, there are a few requirements that he tries to fulfill. As a consequence, the more demands he can fulfill, the higher is the probability that he will keep up the program.

In what concerns physical exercise it was previously shown that self motivation is one of the main issues. As such, fitness equipments must be fun to use and, although high technology is an appellative attribute when choosing between similar equipments, the truth is that they must be simple to use. On the other hand, considering that fitness equipments are to be used at home, they must be both easy to move and must occupy the less space possible. Figure 4 synthesizes the three main requirements that the potential user of a fitness device must fulfill. 


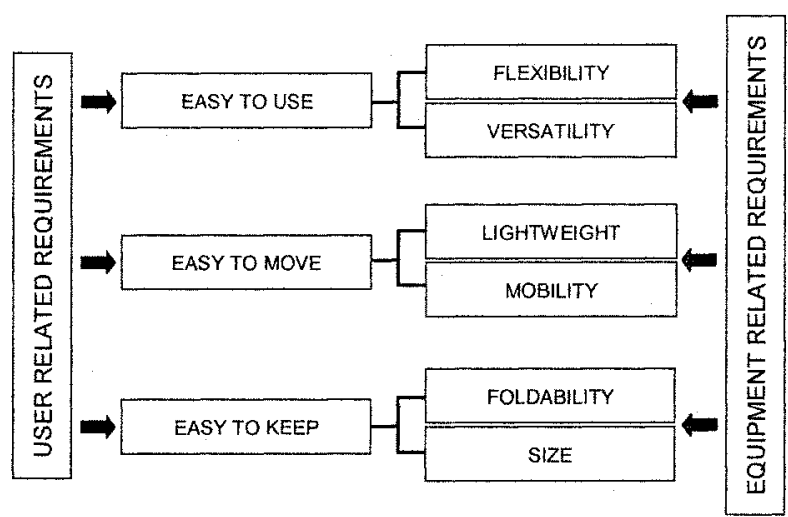

Fig. 4. Home fitness equipment main requirements.

In order to comply with the user's demands, fitness devices must have technical specifications that lead to the satisfaction of the user's preferences and needs. Thus, to be easy to use by any individual, they must present great flexibility to adjust to different persons in a simple and efficient expedite way. They must also be very versatile, allowing their use in many different ways and environments.

The lack of space characterizing the majority of urban buildings makes the ability to be moved and kept in a minimal space two of the major requirements of current fitness equipments. Therefore small equipments are preferred in comparison to larger ones. However, in the latter case, this drawback is minimized by the ability to fold the equipment, which proves to be a good solution. In addition, the weight and mobility of the equipments are intimately related to the need of moving them around the home, between the storing and exercising places.

\subsection{Home Tasks vs. Fitness Devices}

As previously referred, the most common home routine tasks are usually associated to work or leisure. It was also shown that most common work tasks are mainly performed at the kitchen, laundry room or in the home office. When analyzed in more detail, it can be observed that some conventional gait and posture are usually associated to specific home work tasks, like food preparation or serving. It can also be observed that most of these tasks need solely the motion of the upper body, maintaining the lower body completely steady. The movement of the lower body during repetitive tasks would be beneficial for three main reasons: i) reducing the possibility of deficient blood circulation; ii) avoidance of long steady postures and iii) the movement associated to physical and psychological benefits.

Figure 5 shows some of the most usual home routine repetitive work tasks as well as some possible fitness solutions in order to perform simultaneously physical exercise. These tasks were analyzed considering the ergonomic guidelines proposed by Landau et al. [1,2], while design solutions took into consideration the recommendations by Kroemer[3], Panero et al.[4] and the Geneva International Labour Office[5]. 

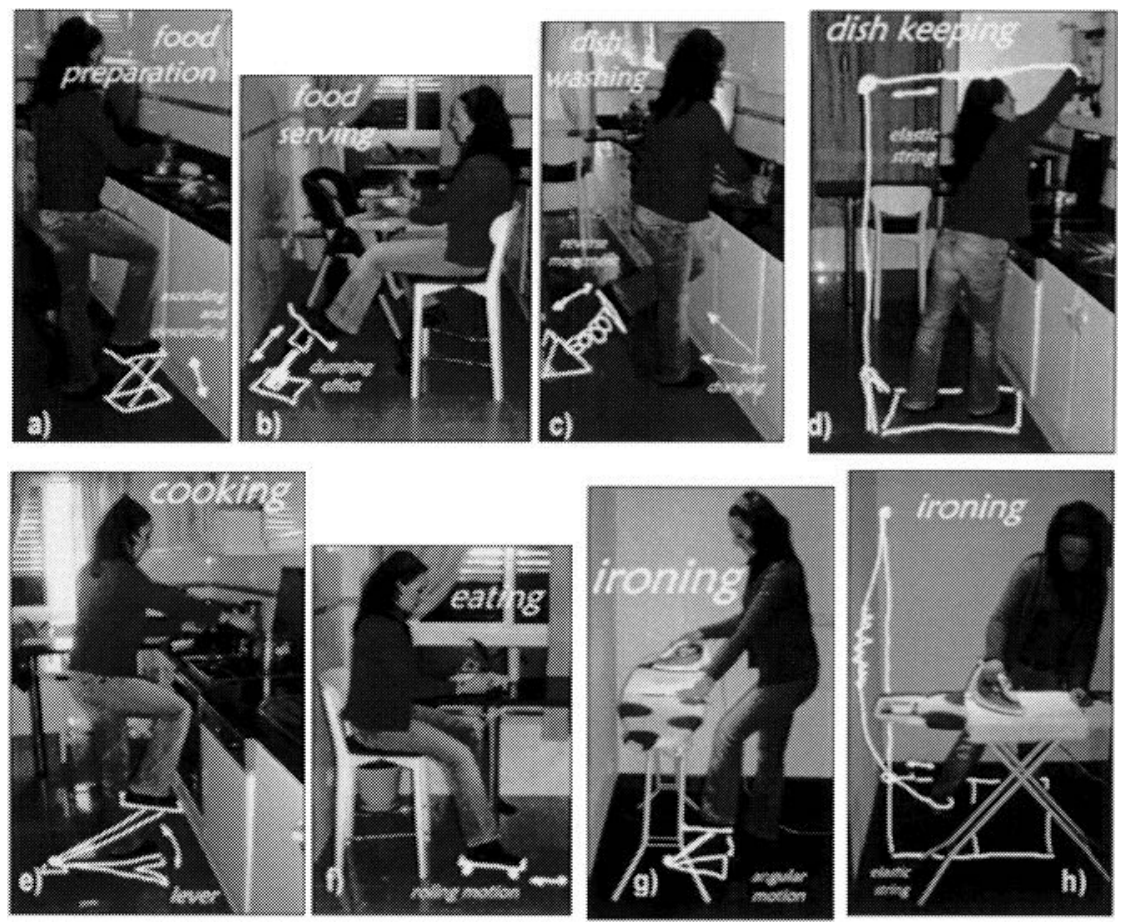

Fig. 5. Home routine work repetitive tasks.

In Figure 6 some of the most usual long leisure situations at home are illustrated. As well as for the home work tasks, some possible fitness solutions are proposed, allowing leisure activities and physical exercise in simultaneous.
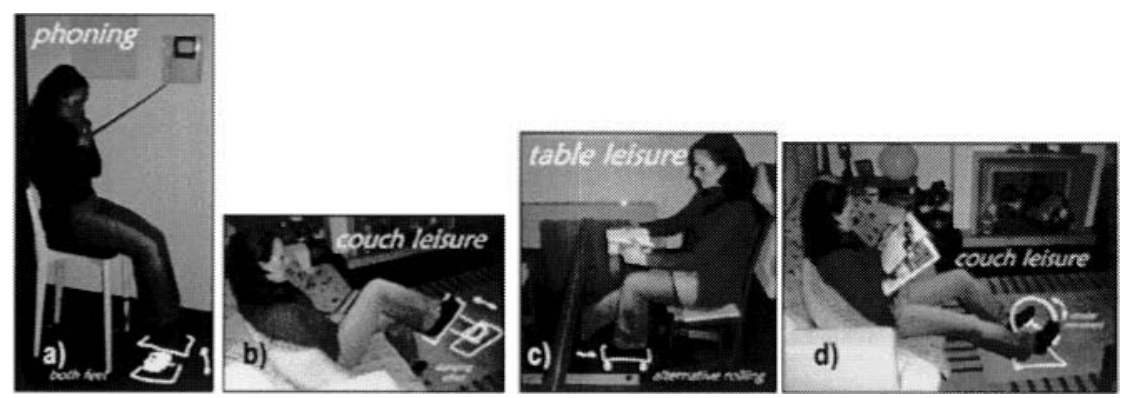

Fig. 6. Home routine leisure tasks. 


\subsection{Resulting Basic Mechanisms}

When considering the design of physical devices from the mechanical point of view, some simple considerations must be taken [6]: the mechanism must be decomposed into functionally independent systems allowing the subsequent generation of concepts.

Observing the different home routine situations of Figures 5 and 6 it is possible to synthesize the main mechanisms involved in the various proposed fitness solutions. Figure 7 summarizes the basic mechanisms presented in Figs. 5 and 6 and their resulting combinations.
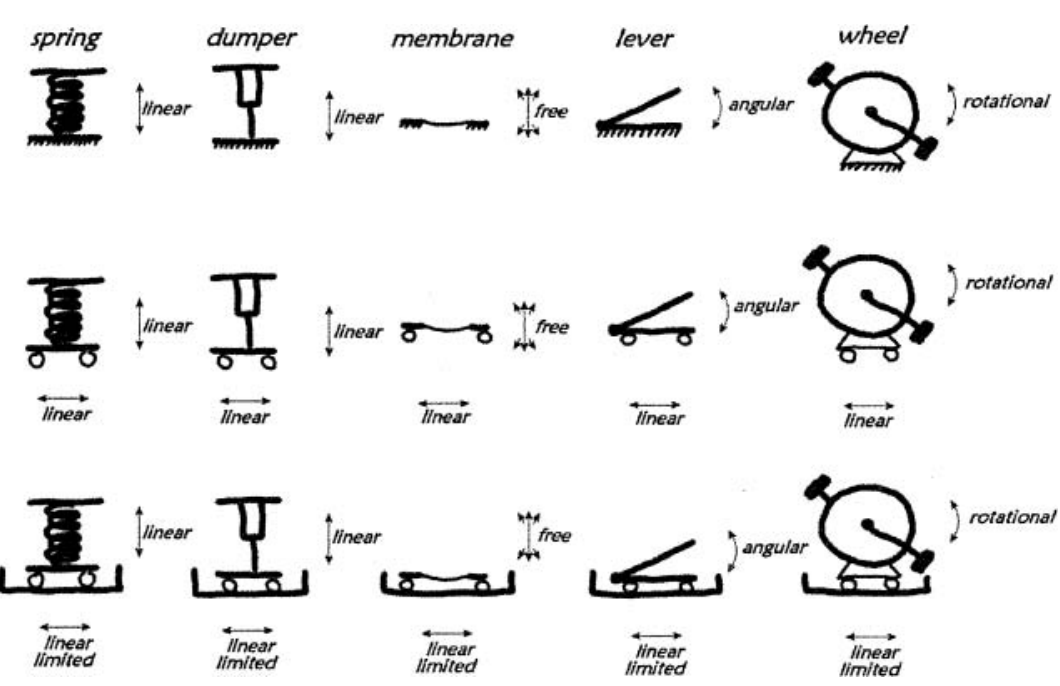

Fig. 7. Basic mechanisms of several home routine fitness solutions.

Home routine fitness solutions base mechanisms.

\section{Designing New Solutions}

The conceptual design phase is one of the most rewarding; yet, it is not free from failure. Considering that one of the major goals of this phase is to spend the least amount of resources in deciding which concepts have the highest potential to solve the desired application problem, attention will be mainly focused on the assumptions presented in previous sections.

Design guidelines [6-8] were considered to enhance the efficiency of the proposed solutions. The concepts presented in the next sections aimed to fulfill the maximum requirements presented before in order to maximize the features in each solution. 


\subsection{Compact Assembling Solution}

From Figures 5 to 7 we can conclude that all solutions, with exception of Figs. 5d) and $5 \mathrm{~h}$ ), are small-sized, being usually lower than $40 \mathrm{~cm}$ height. As a consequence, it can be anticipated that one of the possible concepts focuses on a compact solution that may be easy to carry and to store. Additionally, since versatility is another major requirement, then the ability to have a base structure where different types of exercises can be performed is also very appellative to the potential user.

A linear roller is proposed in the sketch of Figure 8 based on the solutions proposed for the work task of Fig. 5f) and the leisure situation of Fig. 6). The base structure permits not only that the roller gets aligned with the user's foot/leg, but also that it does not roll away from the user. The rolling "gap" may be easily adjusted through simple fasteners, as illustrated in Figure 8. This solution may be used alternatively by each foot, requiring no effort to change the exercised leg. The type of exercise herein proposed may foresee that exercising periods of 10/15 minutes are perfectly acceptable.
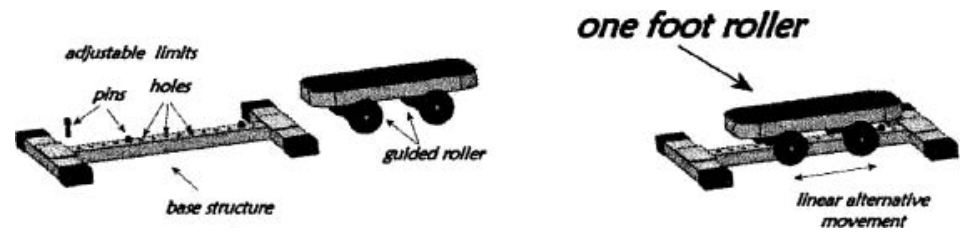

Fig. 8. Sketch representation of a one foot roller exercise device.

In the sketch of Figure 9 a conventional dump-based stepper is proposed in association with a linear roller. This solution is based both on work and leisure tasks, allowing that combined or simple exercises may be performed, which enhances the variety of possible alternative workouts.

The simple grip-components allow a quick and easy fixing of the stepper on the roller, simplifying the intermutability of exercise devices. When the roller is fastened, a pure stepper exercise can be performed, whereas with the roller loose, a combined exercise is possible. 

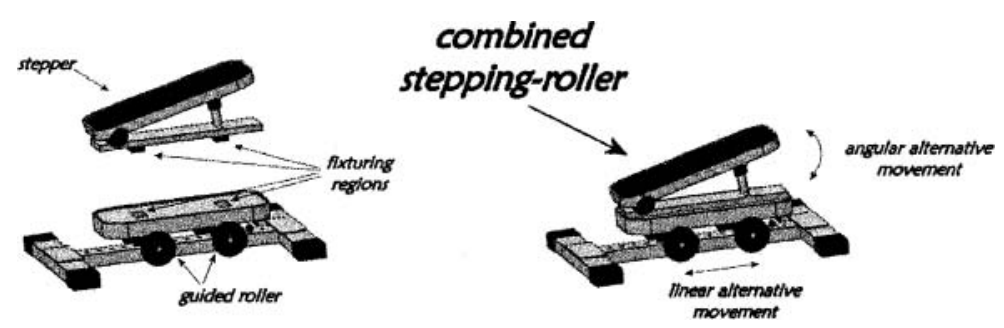

Fig. 9. Sketch representation of a combined stepping-roller.

A compact stationary bike is presented in the sketch of Figure 10 based on the solutions proposed on the leisure situation of Fig. $6 \mathrm{~d}$ ). The base structure permits an easy fixing of the bike pedals. The relative position of the device in the base structure can easily be adjusted to every type and size of the potential users. If necessary, a height adjustment can also be included.

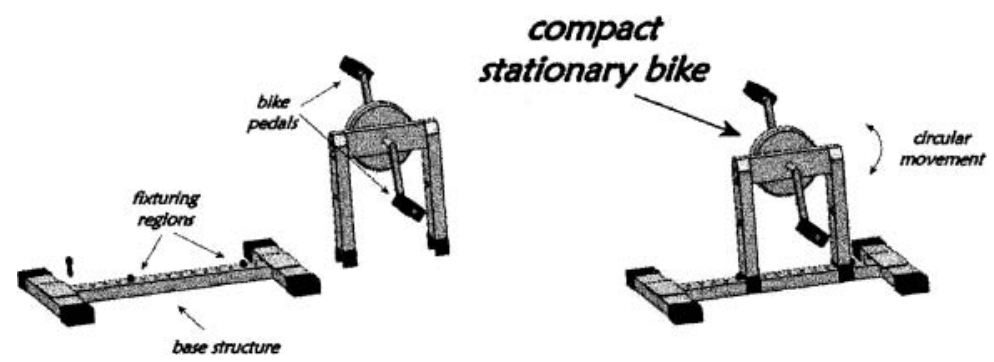

Fig. 10. Sketch representation of a compact stationary bike.

The complete range of devices of the preliminary version for the compact assembling solution can be observed in Figure 11. It includes three main types of exercises and may also allow the inclusion of the remaining mechanisms illustrated in Figure 7. As an example, the inclusion of the membrane stepper can easily be accomplished with the use of a frame to be fitted on the top of the roller. For the horizontal spring and dumper devices, a similar solution can also be considered. 


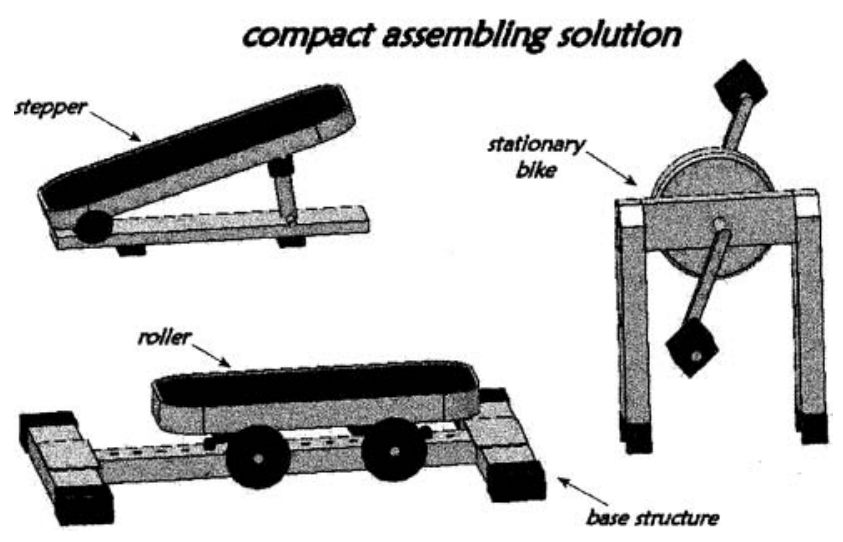

Fig. 11. Home routine exercise devices of the compact assembling solution.

\subsection{Versatile Elastic String Jogger}

When considering elastic-based joggers, three immediate advantages permit selection of that solution on flexible, lightweight trainers. One of the main advantages of a membrane-type exercise device (or of a long elastic string solution) is related to the increase of effort required to reach larger displacements of the elastic components. Unlike what happens when working out with simple weights, where the strength needed to raise the weights remains constant, on elastic components the resistant strength increases with the applied stroke.

The second advantage of the elastic-based joggers is the versatility of movements allowed, imposing no directional restraints, and thus, easier to be used. It can also be easily adapted to almost every type and size of users without major adjustments.

The third, but not the least advantage is related to the ability of working out not only the lower body, but also the upper part of the body. As seen in Figures $5 \mathrm{~d}$ ) and $5 \mathrm{~h}$ ), the simple home routine tasks can be performed with a small effort needed to oppose to the resistant elastic string, when working out not only the lower members, but also the upper members of the body. To improve the ease of use, fast wrist and ankle fixing devices allow a simple and expedite assembling and loosening. Figure 12 illustrates these solutions. 


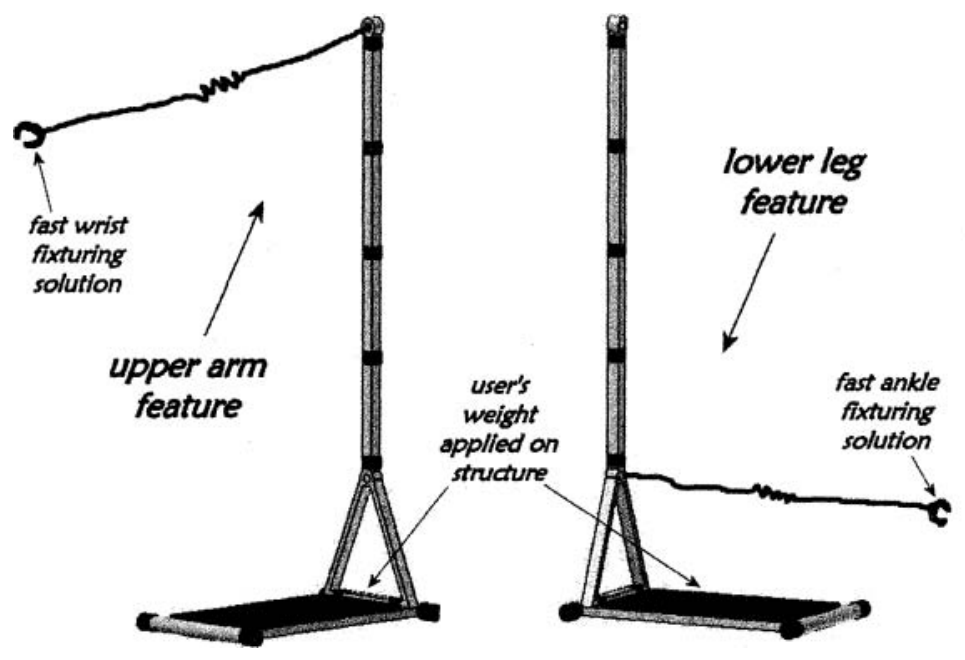

Fig. 12. Upper and lower body exercise solutions on an elastic string jogger.

The complete range of features of the elastic string jogger proposed in this work can be observed in Figure 13. It includes the folding or clamping regions that permit an easy disassembling of the device, as well as the folding joints to pivot the triangular frame. Although it is intended that this device should be very resistant and with a low weight, its ground stabilization is guaranteed by the user's weight. Additional features can also be included, like the anti-slip rubber coating, enhancing the security of use associated to this innovative device." 


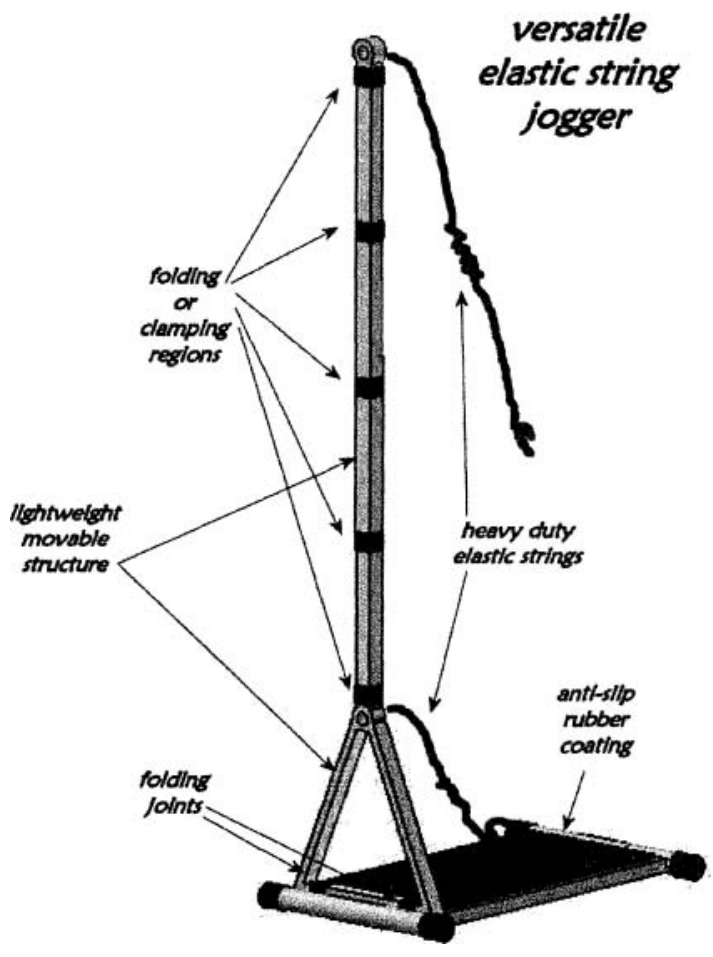

Fig. 13. Sketch view of the versatile elastic string jogger.

\section{Preliminary Conclusions}

The present work points out several solutions to address the problem of motivating people to do exercise regularly and, thus, become healthier. The most usual home routine tasks were studied and different forms of training were considered in order to obtain conceptual designs of innovative fitness solutions. As a result, tailored fitness devices are proposed to allow performing simultaneously home routine tasks and physical exercises. Two preliminary solutions are presented. A compact assembling solution including combined features characteristic of a roller, a stepper and a stationary bike is presented. A versatile elastic string jogger is also presented, allowing physical workout of the user's upper and lower body regions while performing regular home tasks. 


\section{References}

1. Landau, K., Rohmert, W., Brauchler, R.: Task Analysis: Part I. In: Mital, A., Kilbom, A, Kumar, S. (ed.): Ergonomics Guidelines and Problem Solving, Vol. I. Elsevier Science (2000)

2. Brauchler, R., Landau, K.: Task Analysis: Part II. In: Mital, A., Kilbom, A, Kumar, S. (ed.): Ergonomics Guidelines and Problem Solving, Vol. I. Elsevier Science (2000)

3. Kroemer, K.H.E.: Ergonomics: How to Design for Ease and Efficiency. 2nd edn. PrenticeHall (2001)

4. Panero, J., Zelnik, M.: Human Dimension \& Interior Space. Watson Guptil Publications (2002)

5.: I.L.O.: Ergonomic Checkpoints. Geneva International Labour Office (1996)

6. Uliman, D.G.: The Mechanical Design Space. McGraw-Hill (1992)

7. Shigley, J.E., Mishke, C.R.: Mechanical Engineering Design. 5th edn. McGraw-Hill (1989)

8. Norton, R.L: Machine Design. An Integrated Approach. Prentice_Hall (1996) 